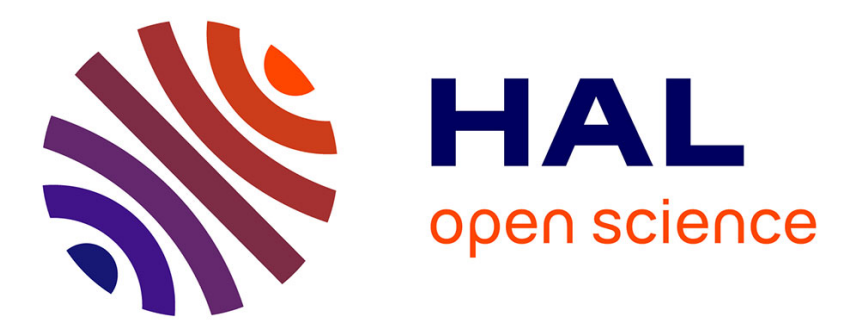

\title{
How Recent Advances in Biology of Waldenström's Macroglobulinemia May Affect Therapy Strategy
}

Marine Baron, Laurence Simon, Stephanie Poulain, Veronique Leblond

\section{To cite this version:}

Marine Baron, Laurence Simon, Stephanie Poulain, Veronique Leblond. How Recent Advances in Biology of Waldenström's Macroglobulinemia May Affect Therapy Strategy. Current Oncology Reports, 2019, 21 (3), pp.27. 10.1007/s11912-019-0768-4 . hal-02274118

\section{HAL Id: hal-02274118 \\ https://hal.sorbonne-universite.fr/hal-02274118}

Submitted on 29 Aug 2019

HAL is a multi-disciplinary open access archive for the deposit and dissemination of scientific research documents, whether they are published or not. The documents may come from teaching and research institutions in France or abroad, or from public or private research centers.
L'archive ouverte pluridisciplinaire HAL, est destinée au dépôt et à la diffusion de documents scientifiques de niveau recherche, publiés ou non, émanant des établissements d'enseignement et de recherche français ou étrangers, des laboratoires publics ou privés. 


\title{
How Recent Advances in Biology of Waldenström's Macroglobulinemia May Affect Therapy Strategy
}

\author{
Marine Baron $^{1} \cdot$ Laurence Simon $^{2} \cdot$ Stéphanie Poulain $^{3,4} \cdot$ Véronique Leblond $^{1}$
}

\begin{abstract}
Purpose of Review Waldenström macroglobulinemia (WM) is a rare lymphoproliferative disorder. Up to now, therapeutic choice was not influenced by the biological characteristics of the disease. Here, we will review how recent advances in biology in WM may affect therapy strategy.

Recent Findings Recently, WM has been described as a new oncogenic model. MyD88 mutation has been described as a key driver mutation and has functional consequences which could be targeted. Other mutations, such as CXCR4 or TP53, have been reported. These mutations are associated with different clinical presentation, prognosis, and treatment response.

Summary Mutational status may influence therapeutic choice in some patients but additional data are required. New targeted therapies are on development.
\end{abstract}

Keywords Waldenström $\cdot$ Personalized treatment $\cdot$ CXCR4 mutation $\cdot$ MyD88 $\cdot$ Next-generation sequencing

\section{Introduction}

Waldenström macroglobulinemia (WM) is a rare lymphoplasmacytic lymphoma characterized by cellular bone marrow (BM) involvement and monoclonal IgM production. In many cases, the diagnosis is fortuitous and there is no need for immediate therapeutic intervention. Criteria for treatment initiation are cytopenia (BM involvement, immunological destruction, or chronic inflammation), constitutional symptoms, hyperviscosity syndrome, and complications related to IgM activity (anti-myelin-associated glycoprotein (MAG) neurop-

Marine Baron

marine.baron@aphp.fr

1 Department of Hematology, Pitié-Salpétrière Hospital APHP, Sorbonne Université, Boulevard de l'hôpital, 75013 Paris, France

2 Department of Hematology, Centre-Hospitalier Sud-Francilien, Corbeil-Essonnes, France

3 Department of Cellular Hematology, CHU de Lille, Lille, France

4 INSERM UMRX 1172, IRCL, Lille, France athy, cold agglutinins disease ...), or to deposits (AL amyloidosis ...) [1].

At the moment, therapeutic choices are guided by patients' characteristics (age, general condition, and comorbidities) and urgent efficacy need but not by biological disease's characteristics [2]. Most of the patients are initially treated with immunochemotherapies with rituximabbased regimens.

Recently, various recurrent mutations, including MyD88 and CXCXR4 mutations, have been described with different clinical presentations, prognoses, and responses to treatment $[3,4]$. These mutations could influence future therapeutic choices. Furthermore, the functional consequences of those mutations could be targeted and new therapies are on development. Finally, p53 defective pathways have been reported in various lymphoproliferative disorders, such as chronic lymphocytic leukemia (CLL), and are associated with poor prognosis and poor treatment responses [5]. Targeted therapies, such as Bruton tyrosine kinase (BTK) inhibitors, are used to bypass this p53 defects [6]. In MW, p53 defective pathways and their functional consequences have been very recently reported.

This review highlights how recent advances in biology of MW could affect therapies. 


\section{Body Headings}

\section{MyD88 Mutation: a Diagnosis Tool and Prognostic Factor?}

The $L 265 P$ mutation of myeloid primary differentiation 88 (MyD88) has been first reported in more than $90 \%$ of MW by Treon 6 years ago using whole-genome sequencing and has been rapidly confirmed by other teams $[4,7]$. MyD88 is a signaling adaptive protein that activates the NF- $\mathrm{KB}$ pathway following stimulation with Toll-like receptors (TLRs) and receptors for IL1 and IL18, as described in Fig. 1. Following ligand binding, receptors are associated with MyD88 as a homodimer. Then, the IL1 receptor kinase 4 (IRAK4) is recruited to MyD88 and phosphorylates IRAK1 and IRAK2 to form a complex known as the "myddosome" which activates the NF- $\mathrm{kB}$ pathway and the mitogen-activated protein kinase (MAPK) pathway. $M y D 88$ activating mutation is responsible for enhancement cell survival through increase in NF-KB activity, JAK-STAT3 signaling, and consequently cytokine production. This mutation is not specific of WM and is found, to a lesser extent, in other indolent or aggressive lymphoproliferative disorders [4, 9]. Indeed, MYD88 L265P mutation is detected in nearly half of IgM monoclonal gammopathy of undetermined significance (MGUS) patients, $10 \%$ of splenic marginal zone lymphoma patients, $4 \%$ of CLL patients, one quart of activated $\mathrm{B}$ cell $(\mathrm{ABC})$ diffuse $\mathrm{B}$ cell lymphoma patients (DLBCL), and $70 \%$ of central nervous system (CNS) DLBCL patients $[9,10]$. The mutation is not found in $\operatorname{IgG}$ MGUS and myeloma patients or healthy donors. A highest BM disease burden and a better prognosis were initially reported in $M y D 88$-mutated (mut) versus $M y D 88$ wild-type (WT) patients but these data are still controversial [11-13]. Indeed, Treon showed, in 326 patients, an estimated 10-year survival of $73 \%$ versus $90 \%$, in $M y D 88$ WT versus $M y D 88$ mut patients respectively [13]. But, more recently, no difference in tumor burden or overall survival were found by Mayo Clinic group in 219 patients [11]. This difference can be explained because patients with indolent disease were excluded and overall survival was estimated in symptomatic WM patients only. Indeed, time to progression from smoldering to active disease seemed to be shorter for $M y D 88$ WT patients

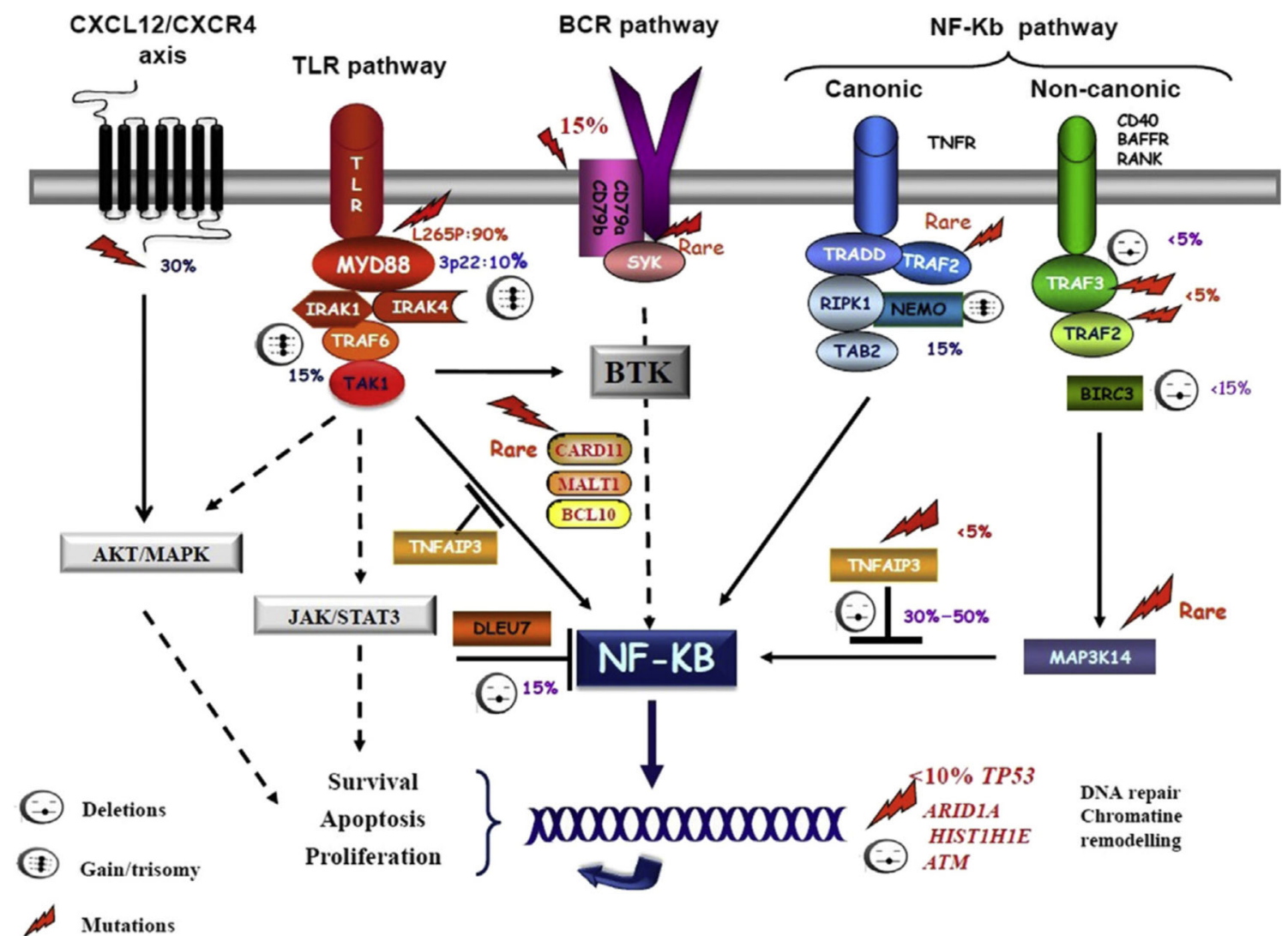

Fig. 1 High-throughput whole-genome sequencing, single nucleotide polymorphism (SNP), and comparative genomic hybridization (CGH) array have identified several pathways characterized with recurrent genomic alterations in WM. Several mechanisms of action are involved in the gene deregulation of key signaling pathways, including deletion, mutation, acquired uniparental disomy (UPD), and gain of chromosome.

A constitutive activation of nuclear factor $-\kappa \mathrm{B}(\mathrm{NF}-\mathrm{\kappa B})$ was demonstrated in WM cells. Genes of canonical and noncanonical NF- $\mathrm{KB}$ pathways, CXCR4, Toll-like receptors (TLR), and B cell receptor (BCR) may be activated deregulation of survival, apoptosis and proliferation, which may lead to WM clone expansion and tumor progression. Reprinted from Magierowicz et al. [8], with permission from Elsevier 
in this latest report (1.8 versus 2.9 years without reaching statistical significance). In summary, more prospective studies are needed to conclude.

\section{MyD88 Mutation: Does It Influence Treatment Response?}

Bruton tyrosine kinase (BTK) is a critical node in B cell receptor (BCR) signaling cascades, mediating the signal from BCR to downstream pathways such as NF- $\mathrm{KB}$, phosphatydilinositol3 kinase (PI3K), AKT, and NFAT. In WM cells with MyD88 L265P, MyD88 is preferentially complexed to phosphorylated BTK (pBTK), yet little complexing is observed in lymphoma cells with WT Myd88 [14]. The level of pBTK is higher in WM cells with MyD88 L265P than in WT Myd88 cells. Ibrutinib is a BTK inhibitor, now commonly used in lymphoproliferative disorders, irrespectively of $M y D 88$ mutation. It reduces the binding strength of BTK to MyD88 L265P [14]. The first report of ibrutinib-based treatment $\mathrm{f}$ in WM patients has demonstrated a great efficacy and suggested worst response rates in MyD88 WT patients but was assessed in only five MyD88 WT patients. Those patients had no major response to ibrutinib $[15 \cdot 16]$. Secondarily, no conclusion have been drawn in the arm C of iNNOVATE study, as only one patient had MYD88 WT genotype [17]. This study evaluated ibrutinib plus rituximab versus placebo plus rituximab for patients with relapsed MW. The MyD88 WT patient obtained a stable disease whereas overall response rate (ORR) was $90 \%$. Recently, Dimopoulos reported a slight lower response rate in $11 M y D 88 \mathrm{WT}$ patients versus 58 mut patients treated with rituximab plus ibrunitib $(81 \%$ of overall response rate versus 97\%) [18]. MyD88 mutational status did not seem to impact rituximab response rate in the rituximab plus placebo group. These minor differences in response rates with regard to the $M y D 88$ mutational status did not affect the progression free survival (PFS). These results may be interpreted with caution in this small series. The question whether $M y D 88$ mutation is a predictive factor in WM patients treated with ibrutinib is still open. Conventional immunochemotherapies should be preferred in case of $M y D 88$ WT pending further information. Few data are available to compare therapeutic responses to standard immunochemotherapies according to mutational status. Paludo et al. reported that regimens with bendamustine and rituximab (BR) or with dexamethasone, rituximab, and cyclophosphamide (DRC) are equally effective in $38 \mathrm{~L} 265 \mathrm{P}$ and 10 WT $M y D 88$ patients with similar response rates and similar time to new treatment [19]. Very recently, FILO French group confirmed that MYD 88 mutations did not impact on disease response for 69 patients treated with BR [20]. We can conclude that $M y D 88$ mutation do not impact on response to conventional chemotherapies.

\section{The Myddosome: Functional Consequences and Targeted Strategies}

New therapeutic approaches targeting $M y D 88$-driven pathway, so-called myddosome (which includes IRAK1/4 and BTK among others), have been developed or are still on development, such as BTK, PI3K, TLR, IRAK, and hematopoietic cell kinase (HCK) inhibitors, as well as targeting the MyD88 molecule itself.

IRAK Inhibitors After stimulation, MyD88 forms complexes with IRAK4. MyD88 L265 P mutation activates downstream signaling pathway, such as IRAK1/4. Indeed, compared with the WT MyD88 protein, the mutated MyD88 protein has enhanced IRAK4 and pIRAK1 binding and can form the myddosome without external stimuli [14, 21]. Furthermore, IRAK1/4 signaling may contribute to persistent WM cell survival following ibrutinib treatment since WM patients cells following 6 months of continued ibrutinib treatment demonstrated highly active IRAK1 and IRAK4, but not BTK [22•]. Treatment of primary WM cells taken from untreated patients, patients on ibrutinib therapy, as well as MYD88-mutated WM cells lines with ibrutinib, and a toolbox IRAK4/IRAK1 inhibitor resulted in more robust reductions in NF- $\mathrm{KB}$ signaling, and at least additive tumor cell killing versus either agent alone [22•]. This study provides a framework for the development and investigation of IRAK inhibitors, alone and in combination with ibrutinib in WM patients.

HCK Inhibitors HCK is a member of the SRC family of protein tyrosine kinases, and one of the most aberrantly upregulated genes in WM cells [23]. HCK is activated by interleukin-6 among others. Yang et al. investigated the role of HCK in $\mathrm{WM}$, and the impact of $M y D 88$ mutation and ibrutinib on the transcriptional regulation and activation of HCK [24•]. They demonstrated that HCK transcription is driven by mutated $M y D 88$ and that HCK is a determinant of survival in MyD88-mutated cells, whereas HCK expression was virtually absent in WT cells. Ibrutinib is also a potent nonconvalent inhibitor of several SRC family members including HCK. Ibrutinib and a preclinical HCK inhibitor attenuated HCK activation and impact survival in Myd88 WM-mutated cells. HCK should represent a novel target for therapeutic development in MyD88-mutated cells.

MyD88 Inhibitors MyD88 dimerization seems to be necessary for assembly of the myddosome, which is composed of MyD88 dimers that recruit and activate IRAK1/2/4 complex and can then trigger NF- $\mathrm{KB}$ activation mediating cell growth and survival signaling. In contrast to native Myd88, mutated MyD88 protein can assemble without external stimuli and trigger constitutive NF-KB activation. First report of $L 265 P$ $M y D 88$ mutation in WM patients has shown marked decrease 
in nuclear staining of NF- $\mathrm{kB}$ p 65 , as well as decreased $\mathrm{IkB} \alpha$ and NF- $\mathrm{KB}$ p 65 phosphorylation after culture of $M y D 88-\mathrm{mu}-$ tated cells with an inhibitor of Myd88 homodimerization [4]. In contrast, WT WM cells were unaffected by the inhibition of MyD88 signaling. More recently, preclinical studies have demonstrated that interference of myddosome assembly within selected regions of the Toll/interleukin-1 receptor (TIR) domain and death domain (DD) of MyD88 protein can impact growth and survival signaling of $M y D 88 L 265 P$-mutated WM cells [25•]. Interference was obtained with mini-peptides designed to compete with MyD88 TIR and DD domain interactions. These findings provide a framework for developing this type of agents.

TLR Inhibitors The $M y D 88$ mutation has been shown to overactivate TLR7 and 9 mediating signaling pathways. Pharmacological inhibitors of TLR9 have been considered and clinical trial has been proposed with an oligonucleotide inhibiting TLRs 7.8 and 9 (IMO-8400) in WM patients but results are not known yet. IMO- 8400 has also been studied in mouse xenografts models, alone or in combination with rituximab. It was well tolerated and exerted potent anti-tumor activity against B cell lymphomas with the MYD88 L265P oncogenic mutation, providing a strong rationale to evaluate this treatment for patients harboring $M y D 88$ mutation [26]. Nevertheless, and unexpectedly, Wang et al. have then reported that interference with TLR9 signaling paradoxically promotes accumulation of $M y D 88$-mutated lymphoplasmablasts in vivo [27]. These conclusions raise the possibility that inhibiting these receptors may be associated with adverse implications and have to be specified.

TCR-Based Immunotherapy Moreover, MyD88 L265P containing peptides can elicit human leukocyte antigen (HLA) class-1 restricted cytotoxic $\mathrm{T}$ cell response, supporting the potential for $\mathrm{T}$ cell receptor (TCR)-based immunotherapy. Indeed, the immune system can recognize, and to some extent, eradicate tumor cells but this anti-tumor response is often inefficient. Antigen-specific immunotherapy holds the potential to induce and boost clinically effective cancer $\mathrm{T}$ cell responses. Tumor-specific neoepitopes can derive from protein-alteration mutational events. Nelde et al. have identified a set of HLA class 1 MyD88 L265P-derived ligands that elicit specific $\mathrm{T}$ cell responses [28]. These data highlight the potential of MyD88 L265P-mutation-specific peptides-based immunotherapy as a novel personalized treatment approach.

\section{CXCR4 Mutation: a Prognostic Factor?}

CXCR4 is a chemokine receptor that promotes WM survival, migration, and adhesion to the BM stroma through interaction with its ligand CXCL12 (or SDF-1) [29]. WM cells express CXCR4. Activating C-X-C chemokine receptor type-4
(CXCR4) frameshift or nonsense mutations in the $\mathrm{C}$ terminal tail was demonstrated in approximately $30 \%$ of WM patients [3]. The location of these somatic mutations is similar to that observed in the germline of patients with warts, hypogammaglobulinemia, infections, and myelokathexis (WHIM) syndrome, a congenital immunodeficiency disorder characterized by chronic noncyclic neutropenia [30]. Importantly, 98\% CXCR4 mut patients are also MyD88 $L 265 P$ positive, suggesting a potential cooperation between these pathways. These activating mutations impair receptor internalization and result in persistent $C X C R 4$ activation and BM myeloid cell trafficking. At least, 40 different $C X C R 4$ somatic mutations have been identified in patients with WM. Furthermore, genes related to $C X C R 4$ signaling were upregulated (CXCR4, CXCL12/SDF-1, and VCAM1), regardless of the $C X C R 4$ mutational status [31]. This confirms the crucial role of $C X C R 4$ pathway in WM pathophysiology. Preclinical studies with the most common CXCR4 S338X mutation in WM have shown sustained signaling of AKT, ERK, and BTK following CXCL12 binding in comparison with wildtype $C X C R 4$, as well as increased cell growth and survival of WM cells. CXCR4 mutations are associated with complex genomic aberrations $[8,32]$. Patients with $C X C R 4$ mutations demonstrate lower adenopathies, delineating differences in disease tropism, and nonsense mutations are associated with higher IgM production and hyperviscosity [12]. Overall survival is not adversely impacted by $C X C R 4$ mutational status $[12,17]$.

\section{CXCR4 Mutation: Does It Influence Treatment Response?}

A potential clinical impact of $C X C R 4$ mutation has been suggested by several teams, by showing drug resistance in vitro $[33 \cdot, 34]$. Indeed, Rocarro and colleagues investigated the functional role of this variant. CXCR4-mutated mice presented with a significant dissemination of tumor cells to distant organs and a reduced survival. WMmutated cells demonstrated resistance to BTK as well as PI3K and $\mathrm{m}$-Tor inhibitors. Importantly, proteasome inhibitors were equally effective in exerting toxicity against both mutated and non-mutated cells. Anti-CXCR4 monoclonal antibodies were effective against WM cells, independently of their mutational status [34]. In the same way, $\mathrm{Cao}$ and colleagues have demonstrated ibrutinib resistance among MW cells engineered with $C X C R 4$ mutation [33•]. These data were in support of initial data indicating that CXCR4-mutated patients presented with resistance to ibrutinib but these findings were based on a small cohort of patients (21 mutated patients) $[15 \cdot, 16]$. Secondarily, no response differences were described in international trial iNNOVATE for 38 mutated patients treated with ibrutinib plus rituximab $[17,18]$. In the same way, 
CXCR4 mutational status did not seem to impact statistically rituximab response rate in the rituximab plus placebo group although patients with MyD88 L265P CXCR4 WHIM genotype had a numerically higher rate of major response. More recently, 30 patients with treatment-naive WM were treated with ibrutinib [35•]. A higher incidence of adenopathies was observed among patients with CXCR4 WT disease $(n=16)$, as described previously. There was a trend for lower IgM level for WT patients but it did not reach statistical significance. Very good partial response rates were higher (31\% versus $7 \%)$, but not statistically significant, in $C X C R 4 \mathrm{WT}$ patients. Very interestingly, the median time to major response was delayed in mutated patients and was 7.3 versus 1.8 months $(p=0.01)$ when compared with WT patients. Similar response kinetics were also previously observed [16, 17]. Finally, the presence of CXCR4 mutation did not impact on disease response in the recent report of the FILO French group about 69 patients treated with BR [20]. More studies are needed to conclude about clinical impact of CXCR4 mutation when starting a treatment. At this moment, therapeutic choice could not be based on CXCR4 mutational status, but ibrutinib should be avoided for mutated patients who need urgent efficacy. Interestingly, the development of BTK or PLCG2 mutations was identified only in WM patients with $C X C R 4$ mutations, supporting genomic instability as the basis of clonal evolution.

\section{CXCR4 Mutation and Targeted Therapy}

Monoclonal antibody therapy is frequently used in WM. Anti-CD20 antibody rituximab is the most commonly used with great efficacy. New monoclonal antibodies have also been shown to be safe and effective, as anti-CXCR4 antibody. Indeed, targeting the CXCR4-CXCL12 axis with CXCR4 antagonist represents an interesting therapeutic approach in WM patients. Ulocuplumab is a fully humanized anti-CXCR4 monoclonal antibody, with halflife longer than the CXCR4 antagonist plerixafor. It has been shown, in preclinical studies, not only to inhibit CXCR4 activation and migration but also to induce apoptosis of CLL cells at nanomolar concentrations, in the presence or absence of stroma support [36]. It has been used in association, in clinical trials for the treatment of relapsed/refractory multiple myeloma and acute myeloid leukemia patients, with encouraging results [37, 38].

In the context of WM, ulocuplumab has been recently studied both in vitro and in vivo and was equally effective in targeting both CXCR4 WHIM and un-mutated WM cells [34]. The use of the antibody led to significant tumor reduction.
A clinical trial examining ibrutinib with ulocuplumab in symptomatic patients with CXCR4 mutations with $\mathrm{WM}$ is ongoing (NCT03225716).

\section{TP53 Alterations: Does It Influence Prognosis and Treatment Response?}

TP53 is located at the chromosome 17p13 locus and encodes for a tumor-suppressor protein P53. P53 is a transcription factor and acts as a critical regulator of cellular proliferation, cell-cycle check-point, DNA repair, and apoptosis. In the past 5 years, numerous genomic studies identified TP53 as the most-frequently mutated gene across a large spectrum of cancer types [39]. In lymphoid malignancies, the frequency of TP53 mutations is lower than in other types of cancer but is widely described [40]. The deletion of the short arm of the chromosome 17 (Del17p) is also responsible of defective p53 pathway. The responsiveness of many tumor cells to anticancer therapy - chemotherapy or irradiation-generally seems to be highly dependent on the apoptosis-inducing ability of p53. For this reason, TP53 mutations and del17p are associated with unfavorable therapeutic responses and poor prognosis in many cancers. These mutations have been well described in numerous lymphoid disorders, including CLL and marginal zone lymphoma (MZL) [41, 42]. BTK inhibitors, such as ibrutinib, can be proposed to bypass p53 defective pathway for the treatment. In patients with treatmentnaïve MW, del17p are encountered in $7 \%$ of patients and are associated with shorter PFS (18.7 months versus 30 months) and shorter disease-free survival (7.8 months versus 28.8 months) [43]. Recently, TP53 mutations incidence and their functional consequences were analyzed in a series of 125 WM patients [44•]. TP53 mutations were observed in $7.3 \%$ of patients and were associated with TP53 deletions in $58.3 \%$ of the cases, suggesting a high correlation between TP53 mutations and del17p and a frequent biallelic inactivation of TP53 in WM. TP53 alterations (mutation, deletion, or uniparental disomy) presented a greater frequency of genomic aberrations compared with TP53 WT. Low frequency of TP53 mutation at diagnosis was confirmed by other groups [45, 46]. Patients with TP53 alterations had a shorter overall survival and a shorter time to progression to a symptomatic disease. The effect of ibrutinib was assessed in vitro among 8 primary WM cells, of which 3 had TP53 mutation. The sensitivity to induction of cell death was similar independently of TP53 mutational status. Very recently, Gustine et al. have also reported 3 patients with TP53 mutation successfully treated with ibrutinib [45]. These data, consistent with observations in other lymphoid disorders, have to be confirmed in vivo with a large number of patients. In case of similar results in vivo, treatment that either bypass the p53 defective pathway (as BTK inhibitors) or reactivate the $\mathrm{p} 53$ protein should be considered for patients with TP53 alterations. Ibrutinib may 
therefore represent an optimal therapeutic option for bypassing the defective TP53 pathway and overcoming chemoresistance in TP53-mutated patients. This has to be confirmed in clinical trials.

At this time, and pending further data, we can conclude that conventional rituximab-based chemotherapies should be preferred in case of $M y D 88$ non-mutated MW or in case of CXCR4 mutation MW with urgent efficacy need.

\section{Conclusion}

Although WM is a chronic lymphoproliferative disorder, it remains an incurable disease with risk of mortality. Rituximab-based regimens with chemotherapy have been the most used treatments and new treatment strategies are needed. From a biological point of view, WM is a new oncogenic model with MYD88 L265P mutation as a key driver. Other mutations such as CXCR4 or TP53 may play a role in disease pathogenesis, prognostication in new frontline therapy era and even underlying mechanisms of drug resistance. A first step toward a personalized medicine in WM is currently studied in clinical trials.

Authors' Contribution MB and LS contributed in writing the manuscript; $\mathrm{VL}$ and SP contributed in revising the manuscript.

\section{Compliance with Ethical Standards}

Conflict of Interest Marine Baron declares that she has no conflict of interest.

Laurence Simon declares that he has no conflict of interest.

Stéphanie Poulain declares that she has no conflict of interest.

Véronique Leblond has received research funding from Roche, and has received compensation from Roche, Janssen, Gilead, AbbVie, and Servier for service as a consultant.

Human and Animal Rights and Informed Consent This article does not contain any studies with human or animal subjects performed by any of the authors.

Publisher's Note Springer Nature remains neutral with regard to jurisdictional claims in published maps and institutional affiliations.

\section{References}

Papers of particular interest, published recently, have been highlighted as:

- Of importance

1. Simon L, Baron M, Leblond V. How we manage patients with Waldenström macroglobulinaemia. Br J Haematol. 2018;181: 737-51. https://doi.org/10.1111/bjh.15202.

2. Leblond V, Kastritis E, Advani R, Ansell SM, Buske C, Castillo JJ, et al. Treatment recommendations from the eighth international workshop on Waldenström's macroglobulinemia. Blood. 2016;128:1321-8.

3. Hunter ZR, Xu L, Yang G, Zhou Y, Liu X, Cao Y, et al. The genomic landscape of Waldenstrom macroglobulinemia is characterized by highly recurring MYD88 and WHIM-like CXCR4 mutations, and small somatic deletions associated with B-cell lymphomagenesis. Blood. 2014;123:1637-46.

4. Treon SP, Xu L, Yang G, Zhou Y, Liu X, Cao Y, et al. MYD88 L265P somatic mutation in Waldenström's macroglobulinemia. $\mathrm{N}$ Engl J Med. 2012;367:826-33.

5. Tessoulin B, Eveillard M, Lok A, Chiron D, Moreau P, Amiot M, et al. p53 dysregulation in B-cell malignancies: more than a single gene in the pathway to hell. Blood Rev. 2017;31:251-9.

6. Farooqui MZH, Valdez J, Martyr S, Aue G, Saba N, Niemann CU, et al. Ibrutinib for previously untreated and relapsed or refractory chronic lymphocytic leukaemia with TP53 aberrations: a phase 2, single-arm trial. Lancet Oncol. 2015;16:169-76.

7. Poulain S, Roumier C, Decambron A, Renneville A, Herbaux C, Bertrand E, et al. MYD88 L265P mutation in Waldenstrom macroglobulinemia. Blood. 2013;121:4504-11.

8. Magierowicz M, Tomowiak C, Leleu X, Poulain S. Working toward a genomic prognostic classification of Waldenström macroglobulinemia: C-X-C chemokine receptor type 4 mutation and beyond. Hematol Oncol Clin North Am. 2018;32:753-63.

9. Xu L, Hunter ZR, Yang G, Zhou Y, Cao Y, Liu X, et al. MYD88 L265P in Waldenström macroglobulinemia, immunoglobulin $\mathrm{M}$ monoclonal gammopathy, and other B-cell lymphoproliferative disorders using conventional and quantitative allele-specific polymerase chain reaction. Blood. 2013;121:2051-8.

10. Yu W, Li J, Chen L. Prognostic value and efficacy evaluation of novel drugs for cytogenetic aberrations in multiple myeloma: a meta-analysis. Int J Clin Exp Med. 2014;7:4051-62.

11. Abeykoon JP, Paludo J, King RL, Ansell SM, Gertz MA, LaPlant BR, et al. MYD88 mutation status does not impact overall survival in Waldenström macroglobulinemia. Am J Hematol. 2018;93:18794.

12. Treon SP, Cao Y, Xu L, Yang G, Liu X, Hunter ZR. Somatic mutations in MYD88 and CXCR4 are determinants of clinical presentation and overall survival in Waldenstrom macroglobulinemia. Blood. 2014;123:2791-6.

13. Treon SP, Gustine J, Xu L, Manning RJ, Tsakmaklis N, Demos M, et al. MYD 88 wild-type Waldenstrom macroglobulinaemia: differential diagnosis, risk of histological transformation, and overall survival. Br J Haematol. 2018;180:374-80.

14. Yang G, Zhou Y, Liu X, Xu L, Cao Y, Manning RJ, et al. A mutation in MYD88 (L265P) supports the survival of lymphoplasmacytic cells by activation of Bruton tyrosine kinase in Waldenström macroglobulinemia. Blood. 2013;122:1222-32.

15. Treon SP, Tripsas CK, Meid K, et al. Ibrutinib in previously treated Waldenström's macroglobulinemia. N Engl J Med. 2015;372: $1430-40$. This is the first prospective study reporting highly activity of ibrutinb for previously treated WM patients. The overall response rate was $90.5 \%$ and responses were highest among patients with $M y D 88^{L 265 P} C X C R 4^{W T}$ patients.

16. Treon SP, Xu L, Hunter Z. MYD 88 mutations and response to ibrutinib in Waldenström's macroglobulinemia. N Engl J Med. 2015;373:584-6.

17. Dimopoulos MA, Trotman J, Tedeschi A, Matous JV, Macdonald $\mathrm{D}$, Tam C, et al. Ibrutinib for patients with rituximab-refractory Waldenström's macroglobulinaemia (iNNOVATE): an open-label substudy of an international, multicentre, phase 3 trial. Lancet Oncol. 2017;18:241-50.

18. Dimopoulos MA, Tedeschi A, Trotman J, García-Sanz R, Macdonald D, Leblond V, et al. Phase 3 trial of ibrutinib plus rituximab in Waldenström's macroglobulinemia. N Engl J Med. 2018;378:2399-410. 
19. Paludo J, Abeykoon JP, Shreders A, Ansell SM, Kumar S, Ailawadhi S, et al. Bendamustine and rituximab (BR) versus dexamethasone, rituximab, and cyclophosphamide (DRC) in patients with Waldenström macroglobulinemia. Ann Hematol. 2018;97: 1417-25.

20. Laribi K, Poulain S, Willems L et al (2018) Bendamustine plus rituximab in newly-diagnosed Waldenstrom macroglobulinemia patients. A study on behalf of the French Innovative Leukemia Organization (FILO). Br J Haematol. In press.

21. Ngo VN, Young RM, Schmitz R, Jhavar S, Xiao W, Lim KH, et al. Oncogenically active MYD88 mutations in human lymphoma. Nature. 2011;470:115-9.

22. Yang G, Liu X, Chen J, et al. Targeting IRAK1/IRAK4 signaling in Waldenstrom's macroglobulinemia. Blood. 2015;126:4004. This abstract reported for the first time that combined BTK and IRAK inhibition led to augmented blockade of NF- $K B$ signaling and enhanced WM cell killing.

23. Gutiérrez NC, Ocio EM, de Las Rivas J, Maiso P, Delgado M, Fermiñán E, et al. Gene expression profiling of B lymphocytes and plasma cells from Waldenström's macroglobulinemia: comparison with expression patterns of the same cell counterparts from chronic lymphocytic leukemia, multiple myeloma and normal individuals. Leukemia. 2007;21:541-9.

24. Yang G, Buhrlage SJ, Tan L, et al. HCK is a survival determinant transactivated by mutated MYD88, and a direct target of ibrutinib. Blood. 2016;127:3237-52. In this study, the authors demonstrated that mutated MYD88 WM cell lines had enhanced HCK transcription and activation and that HCK was activated by IL-6. Docking and pull-down studies showed that ibrutinib targets HCK. These findings presented HCK as a novel target for therapeutic development.

25. Liu X, Hunter ZR, Xu L, et al. Targeting myddosome assembly in Waldenstrom Macroglobulinaemia. Br J Haematol. 2017;177:80813. This short report demonstrated for the first time that disrupting myddosome signaling with mini-peptides designed to compete with MyD88 domain interaction can have clinical relevance.

26. Wang D, Jiang W, Sullivan T, Bhagat L. Novel approach to the potential treatment of patients with B-cell lymphomas harboring the MYD88 L265P mutation: combination treatment with TLR antagonist and rituximab. Blood. 2014;124:508.

27. Wang JQ, Beutler B, Goodnow CC, Horikawa K. Inhibiting TLR9 and other UNC93B1-dependent TLRs paradoxically increases accumulation of MYD88L265P plasmablasts in vivo. Blood. 2016;128:1604-8.

28. Nelde A, Walz JS, Kowalewski DJ, Schuster H, Wolz OO, Peper JK, et al. HLA class I-restricted MYD88 L265P-derived peptides as specific targets for lymphoma immunotherapy. OncoImmunology. 2017;6:e1219825.

29. Ngo HT, Leleu X, Lee J, Jia X, Melhem M, Runnels J, et al. SDF-1/ CXCR4 and VLA-4 interaction regulates homing in Waldenstrom macroglobulinemia. Blood. 2008;112:150-8.

30. Hernandez PA, Gorlin RJ, Lukens JN, Taniuchi S, Bohinjec J, Francois F, et al. Mutations in the chemokine receptor gene CXCR4 are associated with WHIM syndrome, a combined immunodeficiency disease. Nat Genet. 2003;34:70-4.

31. Hunter ZR, Xu L, Yang G, Tsakmaklis N, Vos JM, Liu X, et al. Transcriptome sequencing reveals a profile that corresponds to genomic variants in Waldenström macroglobulinemia. Blood. 2016;128:827-38.

32. Poulain S, Roumier C, Venet-Caillault A, Figeac M, Herbaux C, Marot G, et al. Genomic landscape of CXCR4 mutations in Waldenström macroglobulinemia. Clin Cancer Res. 2016;22: 1480-8.

33. Cao Y, Hunter ZR, Liu X, et al. The WHIM-like CXCR4S338X somatic mutation activates AKT and ERK and promotes resistance to ibrutinib and other agents used in the treatment of Waldenstrom's macroglobulinemia. Leukemia. 2015;29:169-76. In this study, WM cells engineered to express CXCR4 mutation showed decreased apoptotic changes following ibrutibib treatment compared to CXCR4 WT cells. This study suggested therapeutic resistance for $C X C R 4$ mutated cells.

34. Roccaro AM, Sacco A, Jimenez C, Maiso P, Moschetta M, Mishima Y, et al. C1013G/CXCR4 acts as a driver mutation of tumor progression and modulator of drug resistance in lymphoplasmacytic lymphoma. Blood. 2014;123:4120-31.

35. Treon SP, Gustine J, Meid K, et al. Ibrutinib monotherapy in symptomatic, treatment-naïve patients with Waldenström macroglobulinemia. J Clin Oncol. 2018;36(27):2755-61. This is the first clinical prospective trial evaluating ibrutinib activity among 30 naïve WM patients. The findings confirmed that time to response was significantly delayed in patients with CXCR4 mutated disease (1.8 vs 7.3 months).

36. Kashyap MK, Kumar D, Jones H, Amaya-Chanaga CI, Choi MY, Melo-Cardenas J, et al. Ulocuplumab (BMS-936564 / MDX1338): a fully human anti-CXCR4 antibody induces cell death in chronic lymphocytic leukemia mediated through a reactive oxygen speciesdependent pathway. Oncotarget. 2016;7:2809-22.

37. Ghobrial IM, Perez R, Baz R, et al. Phase Ib study of the novel antiCXCR4 antibody ulocuplumab (BMS-936564) in combination with lenalidomide plus low-dose dexamethasone, or with bortezomib plus dexamethasone in subjects with relapsed or refractory multiple myeloma. Blood. 2014;124:3483.

38. Zhang Y, Saavedra E, Tang R, et al. Targeting acute myeloid leukemia with a new CXCR4 antagonist IgG1 antibody (PF06747143)in NOD/SCID mice. Blood. 2015;126:1362.

39. Sabapathy K, Lane DP. Therapeutic targeting of p53: all mutants are equal, but some mutants are more equal than others. Nat Rev Clin Oncol. 2017;15:13-30.

40. Xu-Monette ZY, Medeiros LJ, Li Y, Orlowski RZ, Andreeff M, Bueso-Ramos CE, et al. Dysfunction of the TP53 tumor suppressor gene in lymphoid malignancies. Blood. 2012;119:3668-83.

41. Marinelli M, Peragine N, Di Maio V, et al. Identification of molecular and functional patterns of p53 alterations in chronic lymphocytic leukemia patients in different phases of the disease. Haematologica. 2013;98:371-5.

42. Parry M, Rose-Zerilli MJ, Ljungström V, et al. Genetics and prognostication in splenic marginal zone lymphoma: revelations from deep sequencing. Clin Cancer Res Off J Am Assoc Cancer Res. 2015;21:4174-83.

43. Nguyen-Khac F, Lambert J, Chapiro E, Grelier A, Mould S, Barin $\mathrm{C}$, et al. Chromosomal aberrations and their prognostic value in a series of 174 untreated patients with Waldenström's macroglobulinemia. Haematologica. 2013;98:649-54.

44. Poulain S, Roumier C, Bertrand E, et al. TP53 Mutation and its prognostic significance in Waldenstrom's macroglobulinemia. Clin Cancer Res. 2017;23:6325-35. This is the first report which analyzed the TP53 mutation incidence and genomic features and their functional consequences in MW patients. TP53 mutation was observed in $7.3 \%$ of 125 patients and was highly correlated with deletion $17 \mathrm{p}$. The patients had shorter survival and may benefit from BTK inhibitors.

45. Gustine JN, Tsakmaklis N, Demos MG, Kofides A, Chen JG, Liu $\mathrm{X}$, et al. TP53 mutations are associated with mutated MYD88 and CXCR4, and confer an adverse outcome in Waldenström macroglobulinaemia. Br J Haematol. 2018. https://doi.org/10. 1111/bjh.15560.

46. Varettoni M, Zibellini S, Defrancesco I, Ferretti VV, Rizzo E, Malcovati L, et al. Pattern of somatic mutations in patients with Waldenström macroglobulinemia or IgM monoclonal gammopathy of undetermined significance. Haematologica. 2017;102:2077-85. 